\title{
Explosive nucleosynthesis: nuclear physics impact using neutrino-driven wind simulations
}

\author{
Almudena Arcones ${ }^{* \dagger}$ \\ Department of Physics, University of Basel, Klingelbergstraße 82, 4056, Basel, Switzerland \\ E-mail: a.arcones@unibas.ch
}

\section{Gabriel Martínez-Pinedo}

GSI Helmholtzzentrum für Schwerionenforschung, Planckstr. 1, 64291 Darmstadt, Germany

E-mail: g.martinez@gsi.de

\begin{abstract}
We present nucleosynthesis studies based on hydrodynamical simulations of core-collapse supernovae and their subsequent neutrino-driven winds. Although the conditions found in these simulations are not suitable for the rapid neutron capture (r-process) to produce elements heavier than $\mathrm{A} \sim 130$, this can be solved by artificially increasing the wind entropy. In this way one can mimic the general behavior of an ejecta where the r-process occurs. We study the impact of the long-time dynamical evolution and of the nuclear physics input on the final abundances and show that different nuclear mass models lead to significant variations in the abundances. These differences can be linked to the behaviour of nuclear masses far from stability. In addition, we have analyzed in detail the effect of neutron capture and beta-delayed neutron emission when matter decays back to stability. In all our studied cases, freeze out effects are larger than previously estimated and produce substantial changes in the post freeze out abundances.
\end{abstract}

11th Symposium on Nuclei in the Cosmos, NIC XI

July 19-23, 2010

Heidelberg, Germany

\footnotetext{
${ }^{*}$ Speaker.

${ }^{\dagger}$ Support of Swiss National Science Foundation is acknowledged.
} 


\section{Introduction}

Half of the elements heavier than iron are produced by rapid neutron captures in a yet unknown astrophysical scenario. Galactic chemical evolution models favor core-collapse supernovae, since they occur early and frequently enough to account for the abundances observed in old halo stars and in the solar system [1,2]. Although the necessary conditions to produce heavy elements $(A>130)$ are identified [3] (high entropies, low electron fractions, and short expansion timescales), these are not found in the most recent long-time supernova simulations $[4,5]$. When a supernova explodes, matter surrounding the proto-neutron star is heated by neutrinos and expands very fast reaching sometimes even supersonic velocity [6]. This neutrino-driven wind moves through the early supernova ejecta and eventually collides with it. The interaction of the wind with the slow-moving ejecta results in a wind termination shock or reverse shock where kinetic energy is transformed into internal energy. Therefore, the expansion velocity drops and the temperature (and thus the entropy) increases after the reverse shock. The matter near the proto-neutron star consists mainly of neutrons and protons due to the high temperatures in this region. When a mass element expands, its temperature decreases and neutrons and protons recombine to form alpha particles. At lower temperatures some of the alpha particles can form ${ }^{12} \mathrm{C}$ either by the triple alpha reaction or by the sequence $\alpha(\alpha n, \gamma){ }^{9} \mathrm{Be}(\alpha, n){ }^{12} \mathrm{C}$. The carbon nuclei will capture additional alpha particles (alpha-process) until iron group or even heavier nuclei are produced [7]. The amount of these seed nuclei depends on the entropy and the expansion timescale of the ejecta. Once the formation of ${ }^{12} \mathrm{C}$ nuclei freezes out the remaining neutrons can be captured by the newly formed seed nuclei and the r-process starts.

\section{Supernova simulations and nucleosynthesis networks}

For our nucleosynthesis studies [8] we use trajectories, i.e. density and temperature evolutions, from Ref. [4]. These are long-time hydrodynamical simulations that follow the evolution of the explosion and neutrino-driven winds. Explosions are triggered by neutrinos and their luminosities are parametrized to obtain typical explosion energies. The conditions found in the simulations do not allow the synthesis of heavy r-process elements $(A>130)$ [9]. Therefore, we need to artificially increase the neutron-to-seed ratio (by increasing the entropy by a factor two, which is equivalent to divide the density by a factor of two) in order to produce the third r-process peak. This allows us to study the nucleosynthesis of heavy elements in a typical high-entropy neutrino-driven wind. At high temperatures, the evolution of the composition is followed using a full reaction network [10], which includes nuclei from neutrons and protons to Eu. Reactions with neutral and charged particles were taken from the calculations of the statistical code NON-SMOKER [11] and experimental rates were included (NACRE, [12]) when available. The theoretical weak interaction rates are the same as in Ref. [10]. During the r-process phase, i.e. after charged-particle freeze-out, we use a fully implicit network code that includes photodissociation, neutron capture, beta-decay, and fission. Therefore, it can be used to study the late evolution when matter decays to stability and the neutron density becomes very low. 


\section{Impact of the nuclear physics input on the dynamical r-process}

We investigate the sensitivity of r-process abundances to the combined effects of the longtime dynamical evolution and nuclear physics input and provide a link between the behaviour of nuclear masses far from stability and features in the final abundances. The left panel of Fig. 1 shows the temperature evolution during the r-process phase for the three trajectories used in our calculations: the black line ("unmodified") corresponds to the hydrodynamical simulation with the entropy increased and the reverse shock not changed, in the green dashed line the reverse shock is moved to a temperature of $T_{\mathrm{rs}} \approx 1 \mathrm{GK}$, and for the red dotted line ("no rs") the reverse shock was removed. The abundances resulting from these three evolutions are shown in the right panel of Fig. 1, compared to the solar abundances shown by dots. Notice that the long time evolution has a big impact on the position of the peaks and on the troughs.
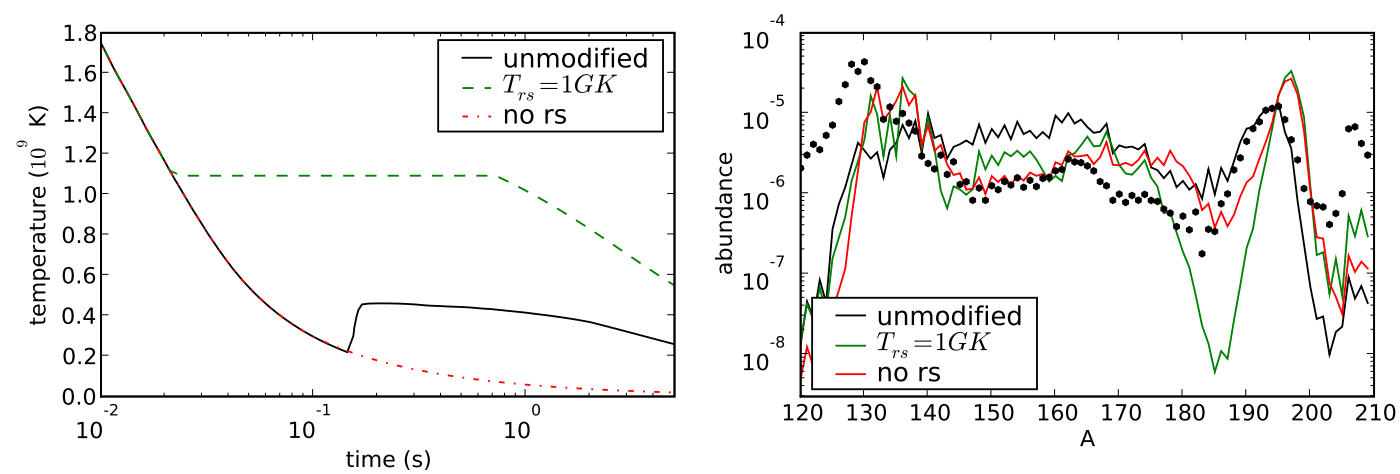

Figure 1: Temperature evolution (left panel) of a mass element ejected at $8 \mathrm{~s}$ after the explosion and variations of the long-time evolution. The right panel shows the final abundances (based on ETFSI-Q mass model) for the three trajectories and the solar abundances by dots.

One can distinguish two typical evolutions depending on the temperature: hot ( $T=1 \mathrm{GK})$ and cold $(T<0.5 \mathrm{GK})$ r-process [13]. In the hot r-process the evolution proceeds under $(n, \gamma)-$ $(\gamma, n)$ equilibrium which lasts until neutrons are exhausted, this is similar to the classical r-process [14]. For the cold r-process, there is a competition between neutron capture and beta decay while photodissociation is negligible. Therefore, the r-process path can move farther away from stability reaching nuclei with shorter half-lives which leads to a faster evolution and an earlier freeze out. Moreover, neutron separation energies have less impact on the final abundances because they enter only through the neutron capture cross section. Notice that photodissociation depends exponentially on the neutron separation energy. The importance of the different nuclear physics input depends thus on the dynamical evolution, therefore all our studies are performed for hot and cold r-process [8].

The sensitivity of the mass model have been investigated by consistently changing neutron separation energies and neutron capture rates for the mass models: FRDM [15], ETFSI-Q [16], HFB-17 [17], and Duflo-Zuker [18]. The final r-process abundances based on these mass models are shown in Fig. 2 for hot and cold r-process conditions. The largest differences in the abundances are in the region around $A \sim 185$ and can be understood looking at the behaviour of the two neutron separation energies before $N=126$ (see Fig. 5 of Ref. [8] which also provides a deeper analysis of the evolution of the abundances). Results based on FRDM are affected by the anomalous behaviour 

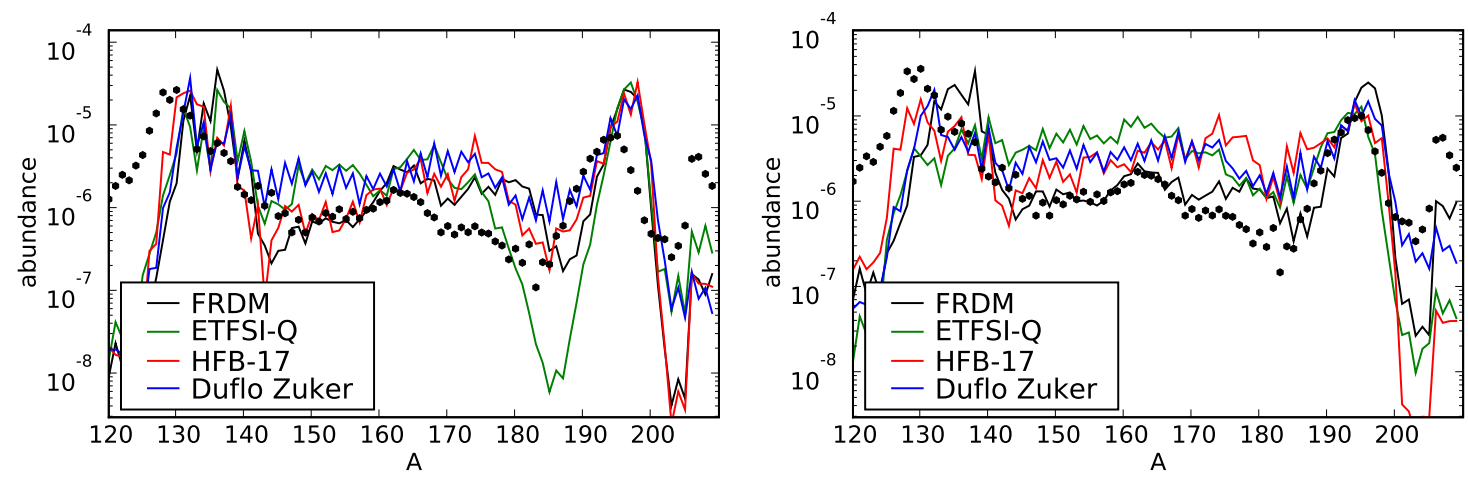

Figure 2: Abundances for the mass models indicated in the caption and for hot (left) and cold (right) rprocess compared to solar (dots).

of the neutron separation energy before $N=90$, which produces the accumulation of matter and thus the formation of peaks around $A \approx 135$ even for the cold r-process (Fig. 2).
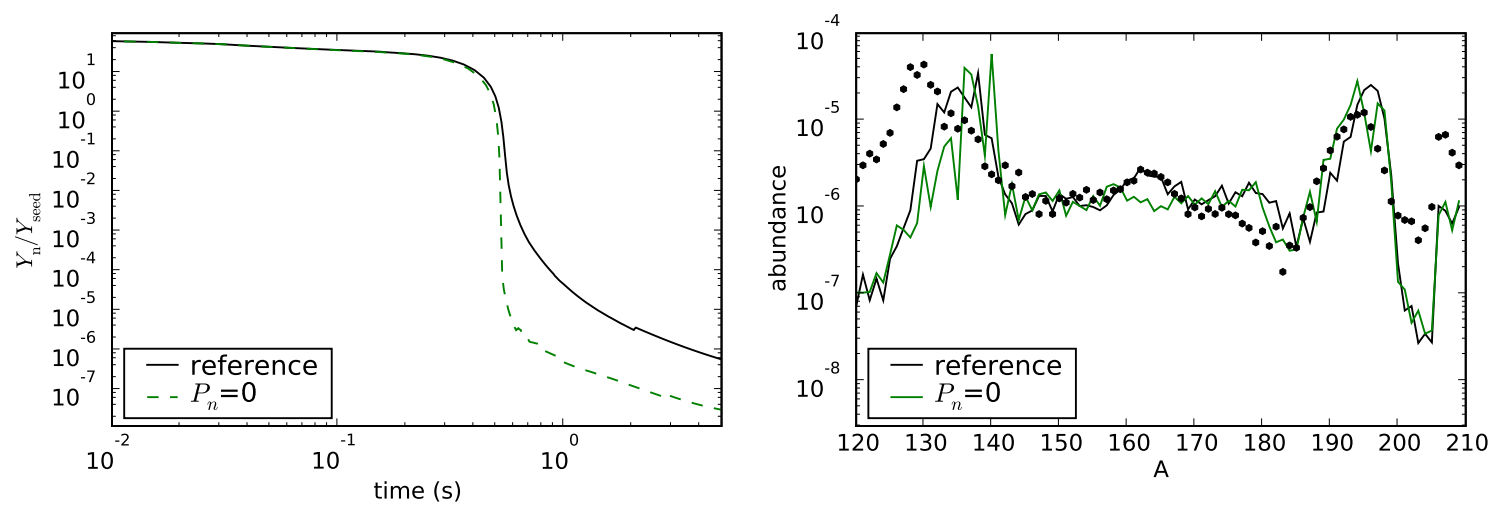

Figure 3: Neutron-to-seed ratio and abundances for the cold r-process. The black lines are for the reference case which is calculated with the standard nuclear input where neutrons are emitted with given probability $\left(P_{n}\right)$ after beta decay. The green lines are for the case where $P_{n}=0$, therefore A is conserved during betadecay.

In dynamical r-process calculations beta-delayed neutron emission and neutron captures contribute to the redistribution of matter, in contrast to the classical r-process calculation (waiting point approximation) where only the first is considered. The neutron captures become very important after freeze out (when neutron-to-seed ratio is around one) as only few neutrons are available and nuclei compete to capture them. We find that the rare earth peak is due to neutron captures when matter moves back to stability, as suggested in Ref. [19]. This implies that the freeze-out of the neutron capture is not instantaneous because neutrons are still needed to form this feature which is present in the solar r-process abundances. Finally, we found that the main contribution of the beta-delayed neutron emission is the supply of neutrons. In our hot r-process calculations, there is almost no difference in the abundance calculated with and without beta-delayed neutron emission because photodissociation prevents the path to reach the regions far from stability where the probability of emitting neutrons after beta decay is higher. In contrast, the suppression of beta-delayed 
neutron emission in cold r-process calculations leads to significant changes in the evolution of the neutron-to-seed ratio. In this case also the neutron-to-seed ratio (shown in the left panel of Fig. 3) reaches very small values which produces a minor shift of the third peak after freeze-out but also inhibits the formation of the rare earth peak (right panel in Fig. 3).

\section{Conclusions}

We have explored the impact of the long-time dynamical evolution and of nuclear masses on the r-process abundances. We have found that the relevance of the different nuclear physics inputs depends on the long-time dynamical evolution [8]. If an $(n, \gamma)-(\gamma, n)$ equilibrium is reached (hot r-process), nuclear masses have a big influence on the final abundances. While for a cold r-process there is a competition between neutron capture and beta decay and these two process become relevant. This rises the importance of future experiments to measure nuclear masses that will provide a direct input for network calculations and constraints for the theoretical mass models. In both cases, as matter decays to stability, neutron captures become key to understand the final abundances and beta-delayed neutron emission becomes important not only for the redistribution of matter, but also for the supply of neutrons. The neutron captures during the decay to stability are required to explain the rare earth peak. More experimental effort is necessary to test the validity of the current theoretical cross sections and more sensitivity studies of the impact of the neutron capture rates on the final abundances will give rise to new insights.

\section{References}

[1] Ishimaru Y., Wanajo S., Aoki W. and Ryan S. G. 2004 ApJL 600 L47-L50

[2] Qian Y. and Wasserburg G. J. 2007 Phys. Rep. 442 237-268

[3] Meyer B. S., Mathews G. J., Howard W. M., Woosley S. E. and Hoffman R. D. 1992 ApJ 399 656-664

[4] Arcones A., Janka H. T. and Scheck L. 2007 A\&A 467 1227-1248

[5] Fischer T., Whitehouse S. C., Mezzacappa A., Thielemann F. and Liebendörfer M. 2010 A\&A 517 A80

[6] Thompson T. A., Burrows A. and Meyer B. S. 2001 ApJ 562 887-908

[7] Woosley S. E. and Hoffman R. D. 1992 ApJ 395 202-239

[8] Arcones A. and Martinez-Pinedo G. 2010 arXiv:1008.3890

[9] Arcones A. and Montes F. 2010 arXiv:1007.1275

[10] Fröhlich C., Martínez-Pinedo G., Liebendörfer M., Thielemann F. K., Bravo E., Hix W. R., Langanke K. and Zinner N. T. 2006 Phys. Rev. Lett. 96142502

[11] Rauscher T. and Thielemann F. K. 2000 At. Data Nucl. Data Tables 75 1-352

[12] Angulo C., et al. 1999 Nucl. Phys. A 656 3-183

[13] Wanajo S. 2007 ApJL 666 L77-L80

[14] Kratz K., Bitouzet J., Thielemann F., Moeller P. and Pfeiffer B. 1993 ApJ 403 216-238

[15] Möller P., Nix J. R., Myers W. D. and Swiatecki W. J. 1995 Atomic Data and Nuclear Data Tables 59185

[16] Pearson J. M., Nayak R. C. and Goriely S. 1996 Phys. Lett. B 387 455-459

[17] Goriely S., Chamel N. and Pearson J. M. 2009 Phys. Rev. C 102152503

[18] Duflo J. and Zuker A. P. 1995 Phys. Rev. C 52 R23-R27

[19] Surman R., Engel J., Bennett J. R. and Meyer B. S. 1997 Phys. Rev. Lett. 79 1809-1812 